\title{
Artigo original \\ Influência da aptidão aeróbia no running anaerobic sprint test (RAST)
}

\author{
Vitor Luiz de Andrade \\ Universidade de São Paulo \\ Carlos Augusto Kalva-Filho \\ Alessandro Moura Zagatto \\ Universidade Estadual Paulista \\ Edson Itaru Kaminagakura \\ Universidade Estadual de Ponta Grossa \\ Marcelo Papoti \\ Paulo Roberto Pereira Santiago \\ Universidade de São Paulo
}

\begin{abstract}
Resumo-O objetivo do estudo foi verificar a possível influência de diferentes níveis de aptidão aeróbia $\left(\mathrm{VO}_{2 \mathrm{MAX}}\right)$ sobre os parâmetros do running anaerobic sprint test (RAST). Para isso, 38 indivíduos (Idade $=18,1 \pm 2,5$ anos, Estatura $=173 \pm 1 \mathrm{~cm}$ e Massa corporal $=65,1 \pm 6,5 \mathrm{~kg}$ ) foram classificados em dois grupos, baixa e elevada aptidão aeróbias (GBA: $\mathrm{n}=22$ e GEA: $\mathrm{n}=16$ ). $\mathrm{O} \mathrm{VO}_{2 \mathrm{MAX}}$ foi determinado por um esforço incremental em esteira rolante até a exaustão voluntária. O RAST foi composto de seis esforços máximos de $35 \mathrm{~m}$ separados por 10 s de intervalo passivo. $\mathrm{O} \mathrm{VO} \mathrm{VMA}_{\text {MA }}$ foi significativamente diferente entre os grupos (GBA $=51,7 \pm 1,9 \mathrm{~mL} \cdot \mathrm{kg}^{-1} \cdot \mathrm{min}^{-1}$; GEA $=58,6 \pm 3,1$ $\left.\mathrm{mL} \cdot \mathrm{kg}^{-1} \cdot \mathrm{min}^{-1}\right)$. A potência média (PM) foi significativamente superior no grupo GBA $(552,7 \pm 132,1 \mathrm{~W})$ em relação ao grupo GEA (463,6 $\pm 132,8 \mathrm{~W}$ ). O impulso (ImP) foi significativamente correlacionado com o $\mathrm{VO}_{2 \mathrm{MAX}}$ no GEA. Pode-se concluir que há um indicativo que o metabolismo aeróbio exerce uma influência na realização do RAST.
\end{abstract}

Palavras-chaves: aptidão aeróbia, aptidão anaeróbia, esportes

\begin{abstract}
Influence of aerobic fitness in running anaerobic sprint test (RAST).” The aim of this study was to investigate the possible influence of different levels of aerobic fitness $\left(\mathrm{VO}_{2 \mathrm{MAX}}\right)$ on the parameters of the running anaerobic sprint test (RAST). Thirty-eight subjects (Age $=18.1 \pm 2.5$ years, Height $=173 \pm 1 \mathrm{~cm}$ and Body mass $=$ $65.1 \pm 6.5 \mathrm{~kg}$ ) were classified into two groups, low and high aerobic fitness (LAF: $\mathrm{n}=22$ and HAF: $\mathrm{n}=16$ ). The $\mathrm{VO}_{2 \mathrm{MAX}}$ was determined by an incremental exercise performed until exhaustion. The RAST was composed of six maximal efforts of $35 \mathrm{~m}$ separated by 10 s passive recovery. The $\mathrm{VO}_{2 \mathrm{MAX}}$ was significantly different between groups $\left(\mathrm{LAF}=51.7 \pm 1.9 \mathrm{~mL} \cdot \mathrm{kg}^{-1} \cdot \mathrm{min}^{-1} ; \mathrm{HAF}=58.6 \pm 3.1 \mathrm{~mL} \cdot \mathrm{kg}^{-1} \cdot \mathrm{min}^{-1}\right)$. The mean power (MP) was significantly higher in the LAF $(552.7 \pm 132.1 \mathrm{~W})$ in relation to the HAF group $(463.6 \pm 132.8 \mathrm{~W})$. The impulse (ImP) was significantly correlated with the $\mathrm{VO}_{2 \mathrm{MAX}}$ in HAF. It can be concluded that there is an indication that the aerobic metabolism exerts an influence on the completion of RAST.
\end{abstract}

Keywords: aerobic fitness, anaerobic fitness, sports

Resumen-“Influencia de la condición aeróbica en el funcionamiento de la prueba de sprint anaeróbico (RAST).” El objetivo de este estudio fue investigar la posible influencia de los diferentes niveles de aptitud aeróbica $\left(\mathrm{VO}_{2 \mathrm{MAX}}\right)$ en los parámetros del running anaerobic sprint test (RAST). Trinta y ocho pacientes (edad = 18,1 $\pm 2,5$ años, altura $=173 \pm 1 \mathrm{~cm}$ y masa corporal = 65,1 $\pm 6,5 \mathrm{~kg}$ ) fueron clasificados en dos grupos, de baja y alta potência aeróbica (BPA: $n=22$ y APA: $n=16$ ). $\mathrm{El} \mathrm{VO}_{2 \mathrm{MAX}}$ se determinó mediante un test de ejercicio progresivo realizado hasta el agotamiento. El RAST se compone de seis esfuerzos máximos de 35m separadas por 10s de recuperación pasiva. El $\mathrm{VO}_{2 \mathrm{MAX}}$ fue significativamente diferente entre los grupos (BPA $=51,7 \pm 1,9 \mathrm{~mL} \cdot \mathrm{kg}^{-1} \cdot \mathrm{min}^{-1}$; APA $=58,6 \pm 3,1 \mathrm{~mL} \cdot \mathrm{kg}^{-}$ $\left.{ }^{1} \cdot \mathrm{min}^{-1}\right)$. La potencia media (MP) fue significativamente mayor en el BPA $(552.7 \pm 132.1 \mathrm{~W})$ en relación con el grupo APA (463.6 $\pm 132.8 \mathrm{~W})$. El impulso (ImP) se correlacionó significativamente con el $\mathrm{VO}_{2 \mathrm{MAX}}$ en APA. Se puede concluir que no hay una indicación de que el metabolismo aeróbico ejerce una influencia sobre la finalización de RAST.

Palabras claves: aptitud aeróbica, aptitud anaeróbica, deportes 


\section{Introdução}

Em esportes coletivos (e.g. basquetebol, futebol, handebol e voleibol) e de raquete (e.g. badminton, críquete, tênis) o padrão de movimento é composto por esforços de alta intensidade separados por períodos de recuperação passiva ou ativa (Castagna, Impellizzeri, Rampinini, D'Ottavio, \& Manzi, 2008; Iaia, Rampinini, \& Bangsbo, 2009). Neste sentido, em situações de alta demanda energética é necessária a interação entre os metabolismos aeróbio e anaeróbio (Glaister, Stone, Stewart, Hughes, \& Moir, 2007). Em esforços de curta duração ( 6s) e alta intensidade o sistema anaeróbio alático promove uma rápida ressíntese de ATP, mantendo assim a disponibilidade de energia para a contração muscular (Hamilton, Nevill, Brooks, \& Williams, 1991). As quantidades de ATP e fosfocreatina (PCr) disponíveis no meio intramuscular são pequenas, e por isso fornecem energia por um curto período (McMahon \& Jenkins, 2002). Desse modo, a recuperação dos estoques destes metabólitos durante a fase de recuperação é fundamental para o desempenho nos esforços subsequentes (Gaitanos, Williams, Boobis, \& Brooks, 1993).

O metabolismo oxidativo é predominante na fase de recuperação após cada esforço intenso, acionando a ressíntese de PCr e a remoção de metabólitos, tais como os fosfatos inorgânicos intracelulares (Pi) (Bailey et al., 2009; Glaister, 2005) e lactato. A recuperação rápida dos estoques de PCr intramusculares é feita nos primeiros instantes de pausa no exercício, que pode ser mensurada pelo componente rápido do consumo de oxigênio (EPOCRAPIDO) (Pan, Hamm, Hetherington, Rothman, \& Shulman, 1991; Sahlin, 1992). Esta fase inicial de decaimento do consumo de oxigênio é significativamente correlacionada com a ressíntese de PCr (Borsheim \& Bahr, 2003). Por este motivo, muito se tem estudado sobre a relação entre a máxima potência aeróbia $\left(\mathrm{VO}_{2 \mathrm{MAX}}\right)$ e a habilidade de manter a desempenho em esforços intermitentes (da Silva, Guglielmo, \& Bishop, 2010; Meckel, Machnai, \& Eliakim, 2009).

Estudos que têm como objetivo determinar as relações entre o $\mathrm{VO}_{2 \mathrm{MAX}}$ e a capacidade de manutenção em esforços repetidos utilizam frequentemente de intervalos entre $20 \mathrm{e}$ 55s (Dupont, McCall, Prieur, Millet, \& Berthoin, 2010; Linossier, Denis, Dormois, Geyssant, \& Lacour, 1993). Estes períodos de recuperação podem não refletir as condições de treinamento e competição de alguns esportes, principalmente esportes coletivos, onde a recuperação ocorre em curtos espaços de tempo (Mohr, Krustrup, \& Bangsbo, 2003; Spencer et al., 2004). Desse modo, ainda são escassos na literatura estudos que investigam a relação entre o $\mathrm{VO}_{2 \mathrm{MAX}} \mathrm{e}$ esforços intermitentes realizados em períodos de recuperação abaixo de 20s. Estudos referentes a este tema em maioria utilizam cicloergômetro para tais análises (Glaister, 2005; Glaister et al., 2007), o que pode não ser considerado específico ao gesto motor envolvido em maioria das modalidades que utiliza a corrida como principal meio de deslocamento. Por isso, o Running Anaerobic Sprint Test (RAST), por sua característica intermitente e mais específica para a avaliação da capacidade de manutenção de esforços repetidos de alta intensidade tem sido muito utilizado em várias modalidades coletivas (Balèiûnas, Stonkus, Abrantes, \& Sampaio, 2006; Moraes \& Pellegrinot, 2006; Spigolon, Borin, Leite, Padovani, \& Padovani, 2007; Roseguini, Silva, \& Gobatto, 2008; Santos, Coledam, \& dos Santos, 2009). Desse modo, a hipótese do estudo é que mesmo em esforços de alta intensidade e curta duração com intervalos pequenos de recuperação pode-se ter uma breve influencia da aptidão aeróbia máxima no desempenho final dos esforços. Por isso, o objetivo do estudo é determinar as possíveis relações entre a potência aeróbia máxima e as variáveis provenientes do RAST em indivíduos com diferentes níveis de aptidão aeróbia.

\section{Método}

\section{Participantes}

Trinta e oito indivíduos participaram voluntariamente do presente estudo (idade $=18,1 \pm 2,5$ anos, estatura $=173 \pm 1 \mathrm{~cm}$ e massa corporal $=65,1 \pm 6,5 \mathrm{~kg}$ ). Somente realizaram os testes aqueles participantes que se manifestaram em Termo de Consentimento Livre e Esclarecido, aprovado pelo Comitê de Ética e Pesquisa da Instituição. Os participantes foram classificados de acordo com os valores de potência aeróbia máxima $\left(\mathrm{VO}_{2 \mathrm{MAX}}\right)$ e atribuídos a dois grupos: 1 ) indivíduos com baixa aptidão aeróbia (GBA; $\left.\mathrm{VO}_{2 \operatorname{MAX}}<55 \mathrm{ml} \mathrm{kg}^{-1} \cdot \mathrm{min}^{-1}\right)$ e 2) indivíduos com elevada aptidão aeróbia (GEA; $\mathrm{VO}_{2 \mathrm{MAX}}$ $>55 \mathrm{ml} . \mathrm{kg}^{-1} \cdot \mathrm{min}^{-1}$ ) (Laursen \& Jenkins, 2002). A divisão foi feita de modo que nenhum atleta do GBA apresentasse maior $\mathrm{VO}_{2 \mathrm{MAX}}$ do que qualquer atleta do grupo GEA (Franchini, Takito, Nakamura, Regazzini, Matsushigue, \& Kiss 1999). Os voluntários GBA (n=22) não participavam de nenhum programa de treinamento padrão e sua frequência de atividade física (i.e. basquete, futebol e corrida) era de três dias por semana. Os indivíduos GEA $(n=16)$ eram engajados em um programa de treinamento periodizado (corrida de fundo e meio fundo) com experiência mínima de um ano e sua frequência de treinamento era de cinco dias por semana, uma vez por dia. A idade entre os grupos não foi significativamente diferente. As caracterís-ticas antropométricas e a diferenciação entre os grupos estão demonstradas na Tabela 1.

\section{Delineamento experimental}

As avaliações foram realizadas em duas visitas. $\mathrm{Na}$ primeira os participantes foram submetidos a um teste incremental até a exaustão voluntária, em esteira rolante para a determinação do $\mathrm{VO}_{\text {2MAx }}$. Na segunda visita os participantes realizaram, em pista de atletismo, seis esforços máximos de 35m separados por intervalo passivo de $10 \mathrm{~s}$ (RAST). As visitas foram separadas por no mínimo 24h e as avaliações foram completadas dentro de sete dias. 
Determinação do máximo consumo de oxigênio $\left(V \mathrm{~V}_{2 \mathrm{MAX}}\right)$

Os valores de $\mathrm{VO}_{2 \mathrm{MAX}}$ foram determinados através de três protocolos incrementais $\mathrm{P} 1(\mathrm{n}=13), \mathrm{P} 2(\mathrm{n}=13)$ e $\mathrm{P} 3(\mathrm{n}=8)$ que se diferenciaram na duração dos estágios (DE; min) e na magnitude dos incrementos (MI; km. $\mathrm{h}^{-1}$ ) (DE x MI; P1: $1 \mathrm{x}$ 0,5; P2: 2x1; P3: 3x1). Nos três protocolos a inclinação se manteve constante $(1 \%)$ e inicialmente os participantes realizaram aquecimento de 5 min a $8 \mathrm{~km} \cdot \mathrm{h}^{-1}$. O VO $\mathrm{VO}_{2 \mathrm{MAX}}$ foi assumido como a maior média de consumo de oxigênio $\left(\mathrm{VO}_{2}\right)$ a cada $15 \mathrm{~s}$.

A diferença entre os protocolos incrementais poderiam influenciar nos valores individuais de $\mathrm{VO}_{2 \mathrm{MAX}}$, no entanto, parece não ocorrer com estas variações de duração (Bentley, Newell, \& Bishop, 2007; Kang, Chaloupka, Mastrangelo, Biren, \& Robertson, 2001). Em todos os casos os participantes atingiram pelo menos dois dos três critérios pra a obtenção do $\mathrm{VO}_{2 \mathrm{MAX}}: 1$ ) Razão de trocas respiratórias $(\mathrm{R}) \geq 1,1 ; 2)$ Frequência cardíaca pico no mínimo ou igual a $90 \%$ da máxima prevista para idade $\left(\mathrm{FC}_{\mathrm{MAx}}=220\right.$-idade) e 3 ) Concentração pico de lactato [Lac] maior que $8 \mathrm{mM}$.

Os testes incrementais foram realizados em esteira rolante (Inbrasport Millennium ATL ${ }^{\circledR}$, Porto Alegre, Brasil) até a exaustão voluntária. A frequência cardíaca (Polar, Modelo T-31, Rio de Janeiro, Brasil), e as variáveis ventilatórias (ParvoMedics TrueOne ${ }^{\circledR} 2400$ Metabolic Measurement System, East Sandy, Utah, USA) foram monitoradas constantemente. As coletas ventilatórias foram realizadas a cada respiração e o analisador de gases foi calibrado automaticamente conforme as especificações do fabricante.

\section{Avaliação do desempenho em esforços intermitentes de alta intensidade}

O desempenho em esforços intermitentes de alta intensidade foi avaliado por meio do RAST. Previamente a realização dos esforços, a massa corporal (MC) total (incluindo vestimentas) foi mensurada por meio de uma balança digital (Tanita UM080, Illinois, USA). O RAST consistiu na realização de seis corridas máximas na distância de $35 \mathrm{~m}$, separadas por um período de recuperação passiva de 10s. O registro de tempo foi realizado a cada esforço (Timex ${ }^{\circledR}$, modelo 85103, São Paulo, Brasil) para a determinação da potência gerada a cada corrida (Potência $=\mathrm{MC}$ x distância ${ }^{2}$ /tempo ${ }^{3}$ ) (Zagatto et al. 2009).

Foram determinadas as seguintes variáveis provenientes do RAST: potência pico (PP), potência média (PM) e o índice de fadiga (IF) $(\mathrm{IF}(\%)=((\mathrm{PP}$ - potência mínima) $\times 100) / \mathrm{PP})$. Por meio da relação entre a distância e o tempo de esforço, foram determinadas a velocidade máxima $\left(\mathrm{V}_{\mathrm{MAX}}\right)$ e a velocidade média $\left(\mathrm{V}_{\mathrm{MED}}\right)$. Além disso, o presente estudo também determinou o impulso gerado durante o RAST (ImP) que foi obtido pela integral resultante da relação entre a força ( $\mathrm{F}=\mathrm{MC} \mathrm{x}$ aceleração) e o tempo de esforço.

\section{Tratamento estatístico}

Os resultados são apresentados em média \pm desvio padrão. Inicialmente, foi aplicado o teste de KolmogorovSmirnov para avaliação da normalidade dos dados, que se apresentaram normais. As diferenças entre os grupos GBA e GEA foram verificadas pelo teste $t$ de student para amostras independentes. As possíveis associações entre os valores de $\mathrm{VO}_{2 \mathrm{MAX}}$ e as variáveis provenientes do RAST foram obtidas através do teste de correlação de Pearson. Todas as análises foram realizadas no software Statística 7 (Statsoft, EUA), e em todos os casos o nível de significância foi préfixado em $95 \%(p<0,05)$.

\section{Resultados}

Os indivíduos atingiram pelo menos dois dos três critérios para obtenção do $\mathrm{VO}_{2 \mathrm{MAX}}$, [Lac] $(8,4 \pm 1,1 \mathrm{mM}), \mathrm{R}$ $(1,3 \pm 0,1 \mathrm{UA})$ e $90 \% \mathrm{FC}_{\text {MAX }}(190 \pm 12 \mathrm{bpm})$.

Tabela 1. Valores médios (MD) \pm desvio padrão (DP) e intervalo de confiança 95\% (IC 95\%) da caracterização da amostra enquadrada em cada grupo.

\begin{tabular}{|c|c|c|c|c|}
\hline & \multicolumn{2}{|c|}{ GBA } & \multicolumn{2}{|c|}{ GEA } \\
\hline & $\mathrm{MD} \pm \mathrm{DP}$ & IC 95\% & $\mathrm{MD} \pm \mathrm{DP}$ & IC 95\% \\
\hline Idade (anos) & $17 \pm 2,3$ & $16,4-18,4$ & $18,9 \pm 2,6$ & $17,5-20,3$ \\
\hline Massa (kg) & $66,0 \pm 6,8$ & $62,7-68,8$ & $64,0 \pm 6,0$ & $61,1-67,5$ \\
\hline Estatura $(\mathrm{cm})$ & $173,0 \pm 5,0$ & $170-175$ & $170 \pm 10,0$ & $169-175$ \\
\hline $\mathrm{VO}_{2 \operatorname{MAX}}\left(\mathrm{L} \cdot \mathrm{min}^{-1}\right)$ & $3,4 \pm 0,3$ & $3,2-3,53$ & $3,8 \pm 0,4^{*}$ & $3,5-4,0$ \\
\hline $\mathrm{VO}_{2 \operatorname{MAX}}\left(\mathrm{ml} \cdot \mathrm{kg}^{-1} \cdot \mathrm{min}^{-1}\right)$ & $51,7 \pm 1,9$ & $50,6-52,7$ & $58,6 \pm 3,1^{*}$ & $56,9-60,2$ \\
\hline
\end{tabular}

* Diferença significativa entre os dois grupos $(\mathrm{p}<0,05)$. 
Tabela 2 . Valores médios (MD) \pm desvio padrão (DP) e intervalo de confiança 95\% (IC 95\%) para variáveis provenientes do RAST. Potência pico (PP), média (PM), velocidade máxima $\left(\mathrm{V}_{\mathrm{MAX}}\right)$ e média $\left(\mathrm{V}_{\mathrm{MED}}\right)$, índice de fadiga (IF) e o impulso (ImP), observados nos grupos, baixa aptidão aeróbia (GBA) e alta aptidão aeróbia (GEA).

\begin{tabular}{|c|c|c|c|c|}
\hline & \multicolumn{2}{|c|}{ GBA } & \multicolumn{2}{|c|}{ GEA } \\
\hline & $\mathrm{MD} \pm \mathrm{PD}$ & IC 95\% & $\mathrm{MD} \pm \mathrm{PD}$ & IC 95\% \\
\hline $\mathrm{PP}(\mathrm{W})$ & $684,2 \pm 172,7$ & $607,7-760,8$ & $595,0 \pm 145,0$ & $517,7-672,2$ \\
\hline (W. $\left.\mathrm{kg}^{-1}\right)$ & $10,3 \pm 2,1$ & $9,4-11,2$ & $9,2 \pm 2,0$ & $8,1-10,3$ \\
\hline $\mathrm{PM}(\mathrm{W})$ & $552,7 \pm 132,1$ & $493,7-611,6$ & $463,6 \pm 132,8^{*}$ & $392,7-534,3$ \\
\hline$\left(\mathrm{W} \cdot \mathrm{kg}^{-1}\right)$ & $8,4 \pm 1,7$ & $7,6-9,1$ & $7,2 \pm 1,8^{*}$ & $6,2-8,1$ \\
\hline $\mathrm{V}_{\operatorname{MAX}}\left(\mathrm{m} \cdot \mathrm{s}^{-1}\right)$ & $7,8 \pm 05$ & $6,8-7,3$ & $6,8 \pm 0,5$ & $6,5-7,8$ \\
\hline $\mathrm{V}_{\mathrm{MED}}\left(\mathrm{m} \cdot \mathrm{s}^{-1}\right)$ & $6,6 \pm 0,4$ & $6,4-6,8$ & $6,2 \pm 0,5^{*}$ & $5,9-6,5$ \\
\hline IF (\%) & $32,6 \pm 10,3$ & $28,1-37,2$ & $37,6 \pm 10,3$ & $32,1-43,1$ \\
\hline $\operatorname{ImP}$ & $2207 \pm 296,5$ & $2075,5-2338,4$ & $2041 \pm 266,7$ & $1900,9-2185,1$ \\
\hline
\end{tabular}

* Diferença significativa entre os dois grupos ( $\mathrm{p}<0,05)$.

Dentre os valores provenientes do RAST, a PM em valores absolutos e relativos à massa corporal e $\mathrm{V}_{\mathrm{MED}}$ foram significativamente diferentes entre os grupos GBA e GEA. A PP em valores absolutos e relativos, a $\mathrm{V}_{\mathrm{MAX}}$, o IF e o ImP não foram diferentes (Tabela 2).

O comportamento do desempenho entre os grupos foi semelhante. No entanto foram encontradas significativas diferenças nos esforços dois $(p=0,02)$ e quatro $(p=0,01)$ que foram maiores no GBA entre os grupos $(p<0,05)$ (Figura 1$)$.

Quando foram analisadas as correlações nos grupos foram encontradas correlações significativas no grupo GBA entre o $\mathrm{VO}_{2 \mathrm{MAX}}$ e a PP (W), a PM (W), IF (\%) e o ImP. No grupo GEA apenas o impulso foi significativamente correlacionado com aptidão aeróbia obtida através do $\mathrm{VO}_{2 \mathrm{MAX}}\left(\mathrm{L} \cdot \mathrm{min}^{-1}\right)$ (Tabela 3).

Tabela 3. Valores de correlação (r) entre o $\mathrm{VO}_{2 \mathrm{MAX}}$ e as variáveis provenientes do RAST.

\begin{tabular}{|c|c|c|c|c|}
\hline & \multicolumn{2}{|c|}{$\mathrm{VO}_{2 \mathrm{MAX}} \mathrm{GBA}$} & \multicolumn{2}{|c|}{$\mathrm{VO}_{2 \mathrm{MAX}} \mathrm{GEA}$} \\
\hline & (L) & $\left(\mathrm{ml} \cdot \mathrm{kg}^{-1} \cdot \mathrm{min}^{-1}\right)$ & (L) & $\left(\mathrm{ml} \cdot \mathrm{kg}^{-1} \cdot \mathrm{min}^{-1}\right)$ \\
\hline PP (W) & $0,43^{*}$ & $-0,45^{*}$ & 0,42 & 0,04 \\
\hline$\left(\mathrm{W} \cdot \mathrm{kg}^{-1}\right)$ & 0,31 & $-0,38$ & 0,40 & 0,02 \\
\hline $\mathrm{PM}(\mathrm{W})$ & 0,17 & $-0,48^{*}$ & 0,16 & $-0,05$ \\
\hline$\left(\mathrm{W} \cdot \mathrm{kg}^{-1}\right)$ & 0,09 & $-0,39$ & 0,17 & $-0,02$ \\
\hline $\mathrm{V}_{\text {MAX }}\left(\mathrm{m} . \mathrm{s}^{-1}\right)$ & 0,14 & $-0,38$ & 0,16 & $-0,02$ \\
\hline $\mathrm{V}_{\text {MED }}\left(\mathrm{m} \cdot \mathrm{s}^{-1}\right)$ & $-0,02$ & $-0,41$ & 0,17 & $-0,03$ \\
\hline IF (\%) & $0,42 *$ & $-0,04$ & $-0,11$ & $-0,11$ \\
\hline $\operatorname{ImP}$ & $0,59 *$ & $-0,51 *$ & $0,65^{*}$ & 0,07 \\
\hline
\end{tabular}

* Diferença significativa entre os dois grupos $(\mathrm{p}<0,05)$. 


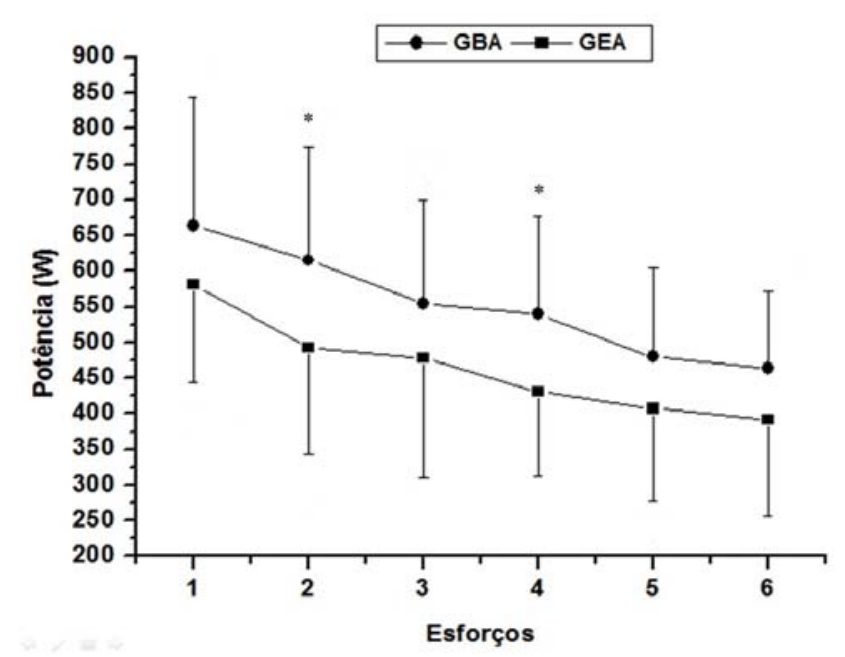

Figura 1. Diferenças entre os esforços do RAST para cada grupo. * Diferença significativa $(p<0,05)$.

\section{Discussão}

O principal achado do estudo foi a confirmação da hipótese de que a aptidão aeróbia máxima $\left(\mathrm{VO}_{2 \mathrm{MAX}}\right)$ pode influenciar os parâmetros provenientes do RAST.

Foi demonstrado na presente investigação que indivíduos treinados aerobiamente possuem maior nível de aptidão aeróbia, no entanto, não possuem diferentes níveis de aptidão anaeróbia (PP). No grupo GBA, em que as atividades físicas realizadas são variadas (i.e. basquetebol, futebol) a capacidade de produzir desempenho anaeróbio não foi diferente do grupo GEA. Possivelmente pela maior adaptação em realizar esforços intermitentes de alta intensidade exigidas pelos esportes envolvidos na prática dos indivíduos do GBA. A PP não foi diferente entre os grupos, no entanto, a PM e $V_{\text {MED }}$ foram significativamente diferentes, indicando uma possível influência da aptidão aeróbia máxima na manutenção dos esforços sucessivos (RAST). O fato do impulso anaeróbio (ImP) ter apresentado correlação significativa em alguns momentos, com o $\mathrm{VO}_{\text {2MAX }}$ reforça essa hipótese.

Em contrapartida, o grupo de GBA obteve melhores níveis médios nos parâmetros do RAST. Nesse sentido, é possível especular a existência de uma relação negativa, entre aptidão aeróbia máxima e aptidão anaeróbia, a qual pode ser reforçada palas correlações negativas observadas no GBA, entre $\mathrm{VO}_{2 \mathrm{MAX}}$ e os valores médios dos esforços no RAST, além disso, a significativa correlação $(r=-0,51)$ encontrada entre o $\mathrm{VO}_{2 \mathrm{MAX}}$ de forma relativa e o ImP reforçam a hipótese de que há uma participação aeróbia durante o RAST, entretanto, ainda não é sabido a magnitude dessa participação.

Mesmo que o $\mathrm{VO}_{2 \mathrm{MAX}}$ apresente importância para rápida recuperação pós-esforço em exercícios máximos de curta duração (Dupont, Millet, Guinhouya, \& Berthoin, 2005), em atividades intermitentes com curtos períodos de recuperação $\left(<20\right.$ s), o $\mathrm{VO}_{2 \mathrm{MAX}}$ parece não ser sensível para influenciar no desempenho. Meckel et al., (2009) encontraram correlações apenas com modelos mais extensos de atividades intermitentes (entre 12 a 20 repetições). Estas correlações parecem ser dependentes de um grande número de repetições e/ou de um período de recuperação maior (>20s) (Aziz, Chia, \& Teh, 2000; Bishop \& Spencer, 2004), o que não ocorre em esforços semelhantes ao RAST. Além disso, têm sido verificadas maiores concentrações de lactato para intervalos menores $(<20$ s) em esforços intermitentes com 10 s de intervalo em comparação aos realizados com 30s de intervalo (Glaister et al., 2007), evidenciando assim uma maior participação da via glicolítica.

De fato á literatura ainda é controversa quanto a influencia do $\mathrm{VO}_{2 \mathrm{MAX}}$ sobre a recuperação em esforços de curta e longa duração (Cooke, Petersen, \& Quinney, 1997; Dupont et al., 2010). Para Castagna et al., (2007) a potência aeróbia pode não ser o melhor indicador recuperação entre os esforços repetidos e curta duração e, dessa forma pode não oferecer um melhor desempenho médio nestes esforços. Em contradição, da Silva et al. (2010) realizaram diversas avaliações em jogadores de futebol bem treinados e não encontraram relação entre a potência aeróbia e desempenho em esforços intermitentes para recuperação de 25s. Esses achados reforçam a idéia de que para recuperações de curta duração $(<20 s)$ o $\mathrm{VO}_{2 \mathrm{MAx}}$ pode não ser o melhor desencadeador de recuperação. da Silva et al., (2010) evidenciaram significativas correlações entre a capacidade aeróbia (LAN) e o desempenho em esforços máximos de 34,2m realizados a cada 25s. Bishop et al. (2004), observaram significativas correlações entre o LAN e o desempenho (5x6s, com 30s de intervalo), não ocorrendo o mesmo em função da intensidade (W). Da mesma forma, Chaovachi et al. (2010) encontraram correlação entre a potência aeróbia obtida pelo Yo-yo Test e esforços intermitentes. Além disso, Edge et al. (2005) realizaram sessões de treinamento intervalado baseado em $\mathrm{VO}_{2 \mathrm{MÁX}}$ e limiar anaeróbio e verificaram melhora no trabalho em esforços intermitentes de até $13 \%$ evidenciando a ligação entre a potência aeróbia e limiar anaeróbio em esforços intermitentes.

Dos modelos fisiológicos de capacidade e potência aeróbias, o $\mathrm{VO}_{2 \mathrm{MAX}}$ é derivado de adaptações centrais (da Silva et al., 2010) e, pode ser limitado se comparado ao limiar anaeróbio (LAN) que é relacionado a fatores periféricos, como o transporte de ions $\mathrm{H}^{+}$e a ressíntese de PCr (Billat, Sirvent, Py, Koralsztein, \& Mercier, 2003), que são considerados essenciais para a manutenção dos esforços intermitentes (Glaister, 2005). Por isso, as fracas correlações encontradas entre os parâmetros do RAST e o $\mathrm{VO}_{2 \mathrm{MAX}}$ podem estar ligadas a uma possível baixa sensibilidade da potência aeróbia máxima em atender demanda fisiológica necessária em esforços sucessivos de alta intensidade. Embora a sensibilidade do $\mathrm{VO}_{2 \mathrm{MAX}}$ seja limitada, parece que o ImP pode ser um bom indicador de recuperação entre os esforços. Entretanto, poucos estudos investigam as correlações entre a LAN e nenhum estudo investigou a influência desses parâmetros no ImP neste tipo de esforço.

Embora os grupos do presente estudo tenham 
apresentado diferença significativa na aptidão aeróbia, a homogeneidade dos dados pode ter sido fator limitante nas correlações e, além disso, outros estudos devem ser realizados para melhor entender a influência entre os parâmetros aeróbios e anaeróbios, como a utilização do LAN e $\mathrm{VO}_{2 \mathrm{MAX}}$. Na necessidade da verificação da fidedignidade do impulso anaeróbio para investigação da queda de rendimento para este esforço bem como a contribuição energética aeróbia e recuperação entre os esforços no RAST ainda devem ser mais bem investigados.

\section{Conclusão}

Pode-se concluir que, pelas diferenças encontradas no desempenho entre os grupos e as fracas correlações com os parâmetros do RAST, o metabolismo aeróbio exerce pouca influência durante o RAST, no entanto, existem evidências de que este metabolismo pode influenciar diretamente em esforços repetidos de alta intensidade, contudo, são necessários estudos que quantifiquem a participação dos metabolismos anaeróbios e aeróbios no RAST.

\section{Referências}

Aziz, A. R., Chia, M., \& Teh, K. C. (2000). The relationship between maximal oxygen uptake and repeated sprint performance indices in held hockey and soccer players. Journal of Sports Medicine and Physical Fitness, 40(3), 195-200.

Balèiûnas, M., Stonkus, S., Abrantes, C., \& Sampaio, J. (2006). Long term effects of different training modalities on power, speed, skill and anaerobic capacity in young male basketball players. Journal of Sports Science and Medicine, 5, 163-170.

Bailey, S. J., Winyard, P., Vanhatalo, A., Blackwell, J. R., DiMenna, F. J., Wilkerson, D. P., . . . Jones, A. M. (2009). Dietary nitrate supplementation reduces the $\mathrm{O}-2$ cost of low-intensity exercise and enhances tolerance to high-intensity exercise in humans. Journal of Applied Physiology, 107(4), 1144-1155. doi: DOI 10.1152/japplphysiol.00722.2009

Bentley, D. J., Newell, J., \& Bishop, D. (2007). Incremental exercise test design and analysis - Implications for performance diagnostics in endurance athletes. Sports Medicine, 37(7), 575586. doi: Doi 10.2165/00007256-200737070-00002

Billat, V. L., Sirvent, P., Py, G., Koralsztein, J. P., \& Mercier, J. (2003). The concept of maximal lactate steady state: a bridge between biochemistry, physiology and sport science. [Research Support, Non-U.S. Gov't Review]. Sports Med, 33(6), 407426.

Bishop, D., \& Spencer, M. (2004). Determinants of repeatedsprint ability in well-trained team-sport athletes and endurancetrained athletes. Journal of Sports Medicine and Physical Fitness, 44(1), 1-7.

Borsheim, E., \& Bahr, R. (2003). Effect of exercise intensity, duration and mode on post-exercise oxygen consumption. [Review]. Sports Med, 33(14), 1037-1060.

Castagna, C., Impellizzeri, F. M., Rampinini, E., D'Ottavio, S., \& Manzi, V. (2008). The Yo-Yo intermittent recovery test in basketball players. [Validation Studies]. Journal of Science and Medicine in Sport, 11(2), 202-208. doi: 10.1016/ j.jsams.2007.02.013

Castagna, C., Manzi, V., D'Ottavio, S., Annino, G., Padua, E., \& Bishop, D. (2007). Relation between maximal aerobic power and the ability to repeat sprints in young basketball players. Journal of Strength and Conditioning Research, 21(4), 11721176.

Chaouachi, A., Manzi, V., Wong del, P., Chaalali, A., Laurencelle, L., Chamari, K., \& Castagna, C. (2010). Intermittent endurance and repeated sprint ability in soccer players. Journal of Strength and Conditioning Research, 24(10), 2663-2669.

Cooke, S. R., Petersen, S. R., \& Quinney, H. A. (1997). The influence of maximal aerobic power on recovery of skeletal muscle following anaerobic exercise. European Journal of Applied Physiology and Occupational Physiology, 75(6), 512519. doi: DOI 10.1007/s004210050197

da Silva, J. F., Guglielmo, L. G. A., \& Bishop, D. (2010). Relationship between Different Measures of Aerobic Fitness and Repeated-Sprint Ability in Elite Soccer Players. Journal of Strength and Conditioning Research, 24(8), 2115-2121. doi: Doi 10.1519/Jsc.0b013e3181e34794

Dupont, G., McCall, A., Prieur, F., Millet, G. P., \& Berthoin, S. (2010). Faster oxygen uptake kinetics during recovery is related to better repeated sprinting ability. European Journal of Applied Physiology, 110(3), 627-634. doi: DOI 10.1007/s00421010-1494-7

Dupont, G., Millet, G. P., Guinhouya, C., \& Berthoin, S. (2005). Relationship between oxygen uptake kinetics and performance in repeated running sprints. European Journal of Applied Physiology, 95(1), 27-34. doi: DOI 10.1007/s00421-005-13828

Edge, J., Bishop, D., Goodman, C., Dawson, B. (2005) Effects of high- and moderate-intensity training on metabolism and repeated sprints. Medicine and Science and Sports Exercise, 37(11), 1975-1982.

Franchini, E., Takito, M.Y., Nakamura, F.Y., Regazzini, M., Matsushigue, K.A., Kiss, M.A.P.M. (1999). Influência da aptidão aeróbia sobre o desempenho em uma tarefa anaeróbia láctica intermitente. Motriz, 5(1), 58-66.

Gaitanos, G. C., Williams, C., Boobis, L. H., \& Brooks, S. (1993). Human muscle metabolism during intermittent maximal exercise. Journal of Applied Physiology, 75(2), 712-719.

Glaister, M. (2005). Multiple sprint work : physiological responses, mechanisms of fatigue and the influence of aerobic fitness. [Review]. Sports Medicine, 35(9), 757-777.

Glaister, M., Stone, M. H., Stewart, A. M., Hughes, M. G., \& Moir, G. L. (2007). The influence of endurance training on multiple sprint cycling performance. Journal of Strength and Conditioning Research, 21(2), 606-612. doi: Doi 10.1519/ 00124278-200705000-00055

Hamilton, A. L., Nevill, M. E., Brooks, S., \& Williams, C. (1991). Physiological responses to maximal intermittent exercise: Differences between endurance-trained runners and games players. Journal of Sports Science, 9(4), 371-382. doi: Pii 785361076 Doi 10.1080/02640419108729897

Iaia, F. M., Rampinini, E., \& Bangsbo, J. (2009). High-Intensity Training in Football. International Journal of Sports Physiology and Performance, 4(3), 291-306.

Kang, J., Chaloupka, E. C., Mastrangelo, M. A., Biren, G. B., \& Robertson, R. J. (2001). Physiological comparisons among three maximal treadmill exercise protocols in trained and untrained individuals. European Journal of Applied Physiology, 84(4), 291-295. doi: DOI 10.1007/s004210000366

Laursen, P. B., \& Jenkins, D. G. (2002). The scientific basis for high-intensity interval training: optimising training programmes and maximising performance in highly trained endurance athletes. [Review]. Sports Medicine, 32(1), 53-73. 
Linossier, M. T., Denis, C., Dormois, D., Geyssant, A., \& Lacour, J. R. (1993). Ergometric and Metabolic Adaptation to a 5-S Sprint Training-Program. European Journal of Applied Physiology and Occupational Physiology, 67(5), 408-414. doi: Doi 10.1007/Bf00376456

McMahon, S., \& Jenkins, D. (2002). Factors affecting the rate of phosphocreatine resynthesis following intense exercise. Sports Medicine, 32(12), 761-784. doi: Doi 10.2165/00007256200232120-00002

Meckel, Y., Machnai, O., \& Eliakim, A. (2009). Relationship among Repeated Sprint Tests, Aerobic Fitness, and Anaerobic Fitness in Elite Adolescent Soccer Players. Journal of Strength and Conditioning Research, 23(1), 163-169. doi: Doi 10.1519/ Jsc.0b013e31818b9651

Mohr, M., Krustrup, P., \& Bangsbo, J. (2003). Match performance of high-standard soccer players with special reference to development of fatigue. [Comparative Study Research Support, Non-U.S. Gov't]. J Sports Sci, 21(7), 519-528. doi: 10.1080/ 0264041031000071182

Moraes, A.M., \& Pellegrinot, I.L. (2006). Evolução da potência dos membros inferiores durante um ciclo de treinamento de pliometria no basquetebol masculino. Revista Digital - Buenos Aires, 10(94), 1.

Pan, J. W., Hamm, J. R., Hetherington, H. P., Rothman, D. L., \& Shulman, R. G. (1991). Correlation of lactate and $\mathrm{pH}$ in human skeletal muscle after exercise by 1H NMR. Magnetic Resonance Medicine, 20(1), 57-65.

Roseguini, A.Z., Silva, A.S., \& Gobatto C.A. (2008). Determinações e Relações dos Parâmetros Anaeróbios do RAST, do Limiar Anaeróbio e da Resposta Lactacidemica Obtida no Inicio, no Intervalo e ao Final de uma Partida Oficial de Handebol. Revista Brasileira de Medicina do Esporte, 14(1), 46-50.

Sahlin, K. (1992). Metabolic factors in fatigue. [Research Support, Non-U.S. Gov't Review]. Sports Medicine, 13(2), 99-107.

Santos, D., Coledam, D.H.C., \& dos Santos, J.W. (2009). Alterações na potência anaeróbia após a pré-temporada em atletas profissionais de futebol. Movimento \& Percepção, 10(15), 93102.

Spencer, M., Lawrence, S., Rechichi, C., Bishop, D., Dawson, B., \& Goodman, C. (2004). Time-motion analysis of elite field hockey, with special reference to repeated-sprint activity. [Comparative Study Research Support, Non-U.S. Gov't]. Journal of Sports Science, 22(9), 843-850. doi: 10.1080/ 02640410410001716715

Spigolon, L.M.P., Borin, J.P., Leite, G.S., Padovani, R.P., \& Padovani, C.R. (2007). Potência anaeróbia em atletas de futebol de campo: diferenças entre categorias. Coleção Pesquisa em Educação Física, 6(1), 421-428.

\section{Nota dos autores}

Vitor Luiz de Andrade e Paulo Roberto Pereira Santiago são filiados à Universidade de São Paulo,- Faculdade de Medicina de Ribeirão Preto, Programa de Pós graduação em Reabilitação e Desempenho Funcional

Carlos Augusto Kalva-Filho é filiado à Universidade Estadual Paulista "Julio de Mesquita Filho" Campus de Presidente Prudente, Departamento de Fisioterapia

Alessandro Moura Zagatto é filiado à Universidade Estadual Paulista "Julio de Mesquita Filho" Campus de Bauru

Departamento de Educação Física

Edson Itaru Kaminagakura é filiado à Universidade Estadual de Ponta Grossa, Departamento de Educação Física

Marcelo Papoti é filiado à Universidade de São Paulo Escola de Educação Física e Esporte de Ribeirão Preto, Laboratório de Biomecânica e Controle Motor

\section{Correspondência}

Vitor Luiz de Andrade

Av. Bandeirantes, 3900, Monte Alegre, Ribeirão Preto,SP 14040-907 Brazil

Fone: +55 16 3602-0529

E-mail: atiedemann@georgeinstitute.org.au

Artigo completo de trabalho apresentado no VIII Congresso Internacional de Educação Física e Motricidade Humana e XIV Simpósio Paulista de Educação Física.

Declaração de Conflito de Interesses: Os autores declararam ausência de conflitos de interesse no que diz respeito à pesquisa, autoria e / ou publicação deste artigo.

Manuscrito recebido em 30 de março de 2013

Manuscrito aceito em 10 de maio de 2013 